\title{
Evaluation of Severity of Poisoning Exposures among Patients Presented to Poison Control Center, Ain Shams University Hospitals, Egypt during 2019
}

\author{
Walaa Gomaa Abdelhamid ${ }^{1}$ \\ ${ }^{1}$ Department of Forensic Medicine and Clinical Toxicology, Faculty of Medicine-Ain Shams University, Cairo Egypt.
}

\begin{abstract}
Background: Poisoning exposure continues to be an important public health concern in many developing countries and a major cause of morbidity and mortality in Egypt. The information released by the Poison Control Center of Ain Shams University Hospitals (PCC-ASUH) is mostly a trigger for increasing hazards challenging the community.

Aim: To evaluate severity of poisoning exposures among patients presented to PCC-ASUH during year 2019.

Methods: A retrospective study involved patients presented to the PCC-ASUH with history of poisoning during 2019.

Results: This study included 21,492 patients. The majority of poisoning incidents were at the age group of 15 - 25 years (34\%) and females outnumbered males (54.8\% versus $45.2 \%$ ). Route of poisoning was mostly oral (89.1\%). Most poison exposures were suicidal (51.5\%). Drug poisoning constituted the majority of cases (59.5\%). Centrally acting drugs were the commonest drugs (18\%) and household chemicals were the commonly used poisons $(13.4 \%)$. Poisoning severity score was mild in $79.4 \%$ of cases, moderate in $14.4 \%$ and severe in $6.2 \%$ of cases. Mortality rate was $0.66 \%$. Higher PSS was associated with prolonged hospital stay and death.

Conclusion: Poisoning is more common in young and middle-aged people. Ingestion was the main route of poisoning and suicidal poisoning predominated. Corrosives and detergents were the most commonly involved toxic substances while centrally acting drugs topped the list of drug poisoning. Higher PSS was associated with prolonged hospital stay and death.

Recommendations:

Poisoning severity score is a simple tool for better poisoning assessment. Strict regulations are needed to control medication marketing.
\end{abstract}

Key words $\quad$ Egypt; Pattern; PCC-ASUH; Poisoning severity score; Suicidal

\section{Introduction}

$\mathrm{P}$ oisoning exposure continues to be an important public health concern in many developing countries and a major cause of morbidity and mortality in Egypt (Tawfik and Khalifa, 2017). Poisoning develops when a toxic substance is ingested, injected, inhaled, or absorbed through the skin, either intentionally or unintentionally (Mowry et al., 2014). Factors affecting the severity and outcome of poisoning in a poisoned patient are multiple and interlinked (Lam, 2003).

Poisoning exposures are increasing day-by-day due to rapid changes in the life style and social behavior (Shadnia et al., 2007). The World Health Organization (WHO) estimated that poisons are responsible for more than one million illnesses worldwide and 0.3 million deaths every year (Thundiyil et al., 2008). Regarding Egypt, the exact total number of poisoning incidents that occur each year is difficult to quantify since most of these events actually go unreported and previous epidemiological studies have focused only on regional data.
Thus recognition of the nature, severity and outcome of acute poisoning cases specific for each country is necessary to determine the characteristics and magnitude of the problem according to which adequate preventive measures and management techniques can be taken (Sahin et al., 2011).

Poison Control Center of Ain Shams University Hospitals (PCC-ASUH) is the major poison control center in Egypt. Since its establishment in 1981, it has provided adequate services in diagnosis and treatment of approximately 23,000 patients annually throughout Egypt. The information released by the PCC-ASUH is mostly a trigger for increasing hazards challenging the community (Tawfik and Khalifa, 2017).

\section{Aim of the Study}

The aim of this study is evaluation of severity of poisoning among patients presented to PCC-ASUH during year 2019, and highlighting different changes in trend of this problem as regards the collected data. Annual reports are essential for interpretation of 
poisoning data to identify continuously emerging hazards and issue warning reports, thus reducing morbidity and mortality of poisoning exposures.

\section{Patients and Methods}

\section{Study design:}

This was a retrospective hospital-based study involved all patients presented to the PCC-ASUH with history of acute poisoning from January to December 2019.

Study setting:

The data had been collected from electronic database and medical records of PCC-ASUH. The study variables included demographics (age, sex and residence); type; route and mode of the poison taken; severity; place and duration of admission; management of poisoning and outcome. Poisoning Severity Score (PSS) of European Association of Poisons Centers and Clinical Toxicologists (EAPCCT) was used to grade severity of poisoning at the time of peak manifestations as regards the patient's clinical features (Persson et al., 1998). Grading was described as: (0): None, no symptoms or signs related to poisoning, (1): Mild, transient and spontaneously resolving symptoms, (2): Moderate, Pronounced or prolonged symptoms, (3): Severe or life-threatening symptoms and (4): Death. The collected data were subjected to statistical analysis and tabulation using SPSS program, version 20. Chisquare test was used to test the association between qualitative variables.

Ethical considerations:

Administrative approval was obtained from PCC-ASUH. The study was approved by the Institutional Review Boards of Faculty of Medicine, Ain Shams University. Patient consent was waived because this was a retrospective study based on medical records. Confidentiality of data was maintained and used only for the purpose of epidemiological analysis.

\section{Results}

During the study period, a total of 21,492 patients of acute poisoning attended the ED of PCCASUH. Age distribution revealed that the majority of poisoning incidents were at the age group of $15-25$ years accounting for $34 \%$ of total cases followed by the age group less than seven years $(26.4 \%)$ while those between seven and 15 years were the least presentable age group $(5.9 \%)$ (Table 1). Incidence of toxic exposure was more common among females compared to males (54.8\% versus $45.2 \%$ ) (Table 2). Most of cases originated from Cairo $(66.5 \%)$ followed by Kalioubeya (18.2\%) and Giza (8.1\%) governorates (Table 3).

Route of poisoning was mostly oral in $89.1 \%$ of cases. Other routes, including inhalation, injection and skin exposures, did not exceed $10.9 \%$ of cases (Table 4). Table (5) showed that suicidal mode of poisoning represented $51.5 \%$ of cases followed by accidental mode, accounting for $39.6 \%$ of cases. Drug overdose, therapeutic errors and criminal causes constituted $8.9 \%$ of cases.
Pharmaceuticals and drugs of abuse were responsible for $59.5 \%$ of poisoning incidents while non-drug poisoning represented $40.5 \%$ of cases (Table 6). Drug poisoning was remarkably due to centrally acting drugs (18\%), where antidepressants predominated $(4.6 \%)$. Drugs of abuse were responsible for $7.9 \%$ of poisoning exposures where cannabis represented the majority of cases $(4.35 \%)$ followed by opiates (1.78\%) (Table 7).

Regarding non-drug poisoning, the highest percentage was related to household chemicals (13.4\%), mainly corrosives which comprised $6.49 \%$ of recorded cases while insecticides and rodenticides accounted for $12.29 \%$ of cases. Cases presented with food poisoning were $7.7 \%$ while animal poisoning was seen in $2.25 \%$ of cases. However toxic gases poisoned cases were $2.32 \%$ which involved mainly carbon monoxide poisoning (Table 8). Table (9) and figure (1) summarized the top ten most frequently involved exposure substances received in PCC-ASUH during the year 2019.

Poisoning severity was mild in the majority $(79.4 \%)$ of cases, moderate in $(14.4 \%)$ and severe in $(6.2 \%)$ (Table 10$)$. Table (11) showed that the majority of studied patients $(79.4 \%)$ needed only observation for a period less than six hours while $14.5 \%$ stayed for six hours to one day and $5.4 \%$ of cases were admitted for more than one day up to four days. Only $0.3 \%$ stayed in the hospital for more than 7 days. Most of studied patients $(97.2 \%)$ were discharged after complete improvement while $2.15 \%$ were discharged against medical advice and death occurred in 143 cases $(0.66 \%)$. (Table 12$)$ The leading causes of death were mainly organophosphates (34 cases), followed by opiates (21 cases), aluminium phosphide (20 cases) and methanol (9 cases) (Figure 2). Other causes of death from different poisons were displayed in Table (13).

Table (14) outlined the use of emergency measures in the studied poisoned patients where $1.24 \%$ of cases required urgent endotracheal intubation and $1.19 \%$ of cases were mechanically ventilated. Dopamine was indicated in $0.27 \%$ of cases. Decontamination procedures were accomplished on $2 \%$ of cases either in the form of gastric lavage or skin wash. Enhanced elimination in the form of activated charcoal was administered for $42.3 \%$ of cases while hemodialysis was the mainstay treatment of 22 cases $(0.1 \%)$. Antidotes treatment accounted for $9.65 \%$ of cases. Atropine and obidoxime were the most common antidotes used followed by naloxone, then snake and scorpion anti venoms.

On studying the association between types of toxic agents and age shown in table (15), there were statistically significant differences between different age groups where household chemicals were the most prevalent types in poisoned cases $<7$ years $(35.9 \%)$ followed by therapeutic drugs (34\%) and insecticides $(12.5 \%)$. Otherwise, insecticides were the most prevalent type in the age group from $7-<15$ years $(27.9 \%)$ followed by drugs $(24.2 \%)$. On the other hand, drug poisoning was more prevalent in the age groups from $15-<25$ years $(60.9 \%), 25-40$ years $(64.7 \%)$ and 
$>40$ years $(49.8 \%)$. The table also revealed that patients from $15-<25$ years were mostly affected by drugs of abuse compared to other age groups.

There was a statistically significant difference as regards types of toxic agents with $\operatorname{sex}(\mathrm{P}=0.000)$ where drugs were the prevalent type of poisoning in males (44.8\%) followed by substance abuse (12.1\%). Regarding females, drug poisoning also predominated $(57.3 \%)$ followed by household chemicals $(16.1 \%)$ as shown in table (16).

Table (17) showed a significant difference regarding age when compared to PSS, period of hospitalization and outcome where increasing age was associated with higher PSS, prolonged hospital stay and higher mortality rate. No significant difference had been observed between males and females regarding PSS, period of hospitalization and outcome as shown in table (18).
A significant difference was found between PSS and length of stay where $4.2 \%$ of patients with PSS (3) were admitted for a period exceeding seven days and only $0.4 \%$ needed observation for a period less than six hours. Mortality rate was significantly higher in patients with PSS (3) compared to those with PSS (1) ( $7.8 \%$ and $0.01 \%$ respectively) (Table 19$)$. In addition, there was a significant relation between period of hospitalization and mortality (Table 20, Figure 3 ).

Regarding toxic agents responsible for mortality in the studied patients, table (21) showed a significant difference between survivors and non-survivors observed with several toxins (e.g. organophosphates, opiates, aluminium phosphide, methanol, corrosives and psychotropics). No significant difference was observed with other toxins (e.g. tramadol, carbon monoxide, zinc phosphide and carbamazepine).

Table (1): Age distribution of poisoned cases received in PCC-ASUH during the year 2019

\begin{tabular}{|c|c|c|}
\hline Age (years) & $\mathbf{N}$ & $\mathbf{\%}$ \\
\hline$<\mathbf{7}$ & 5666 & 26.4 \\
\hline $\mathbf{7}-<\mathbf{1 5}$ & 1269 & 3.9 \\
\hline $\mathbf{1 5}-<\mathbf{2 5}$ & 7289 & 25.3 \\
\hline $\mathbf{2 5}-\mathbf{4 0}$ & 5452 & 8.4 \\
\hline$>\mathbf{4 0}$ & 1816 & 100 \\
\hline
\end{tabular}

N: Number of patients, \%: Percentage

Table (2): Sex distribution of poisoned cases received in PCC-ASUH during the year 2019

\begin{tabular}{|c|c|c|}
\hline Sex & $\mathbf{N}$ & $\mathbf{\%}$ \\
\hline Female & 11771 & 54.8 \\
\hline Male & 9721 & 45.2 \\
\hline Total & 21492 & 100 \\
\hline
\end{tabular}

N: Number of patients \%: Percentage

Table (3): Residence distribution of poisoned cases received in PCC-ASUH during the year 2019

\begin{tabular}{|c|c|c|}
\hline Region & $\mathbf{N}$ & $\mathbf{\%}$ \\
\hline Cairo & 14285 & 66.5 \\
\hline Kalioubeya & 3911 & 18.2 \\
\hline Giza & 1747 & 8.1 \\
\hline Upper Egypt Governorates & 780 & 3.6 \\
\hline Other Delta Governorates & 429 & 2 \\
\hline Suez Canal Governorates & 285 & 1.3 \\
\hline Sina & 55 & 0.3 \\
\hline Total & 21492 & 100 \\
\hline
\end{tabular}

N: Number of patients

\%: Percentage

Table (4): Routes of poisoning in poisoned cases received in PCC-ASUH through year 2019

\begin{tabular}{|c|c|c|}
\hline Route & $\mathbf{N}$ & $\mathbf{\%}$ \\
\hline Ingestion / Oral & 19157 & 89.1 \\
\hline Inhalation / nasal & 1556 & 7.2 \\
\hline Bite / sting & 483 & 2.2 \\
\hline Injection & 210 & 1 \\
\hline Dermal / skin & 86 & 0.4 \\
\hline Total & 21492 & 100 \\
\hline
\end{tabular}

N: Number of patients, \%: Percentage 
Table (5): Modes of poisoning in poisoned cases received in PCC-ASUH during the year 2019

\begin{tabular}{|c|c|c|}
\hline Mode & $\mathbf{N}$ & $\mathbf{\%}$ \\
\hline Suicidal & 11063 & 51.5 \\
\hline Accidental & 8521 & 39.6 \\
\hline Overdose by drug of abuse & 1808 & 8.4 \\
\hline Therapeutic error & 80 & 0.4 \\
\hline Criminal & 20 & 0.1 \\
\hline Total & 21492 & 100 \\
\hline
\end{tabular}

N: Number of patients, \%: Percentage

Table (6): Type of causative agents in poisoned cases received in PCC-ASUH during the year 2019

\begin{tabular}{|c|c|c|}
\hline Type & $\mathbf{N}$ & \% \\
\hline Drug poisoning & 12797 & 59.5 \\
\hline Non- drug poisoning & 8695 & 40.5 \\
\hline Total & 21492 & 100 \\
\hline
\end{tabular}

N: Number of patients, \%: Percentage

Table (7): Pharmaceuticals and drug of abuse intoxicated cases received in PCC-ASUH during the year 2019

\begin{tabular}{|c|c|c|}
\hline Drug & $\mathbf{N}$ & $\%$ \\
\hline Addiction drugs & 1693 & 7.9 \\
\hline Cannabis & 936 & 4.35 \\
\hline Opiates & 382 & 1.78 \\
\hline Tramadol & 245 & 1.14 \\
\hline Strox & 100 & 0.46 \\
\hline Vodoo & 30 & 0.14 \\
\hline Centrally acting drugs & 3874 & 18 \\
\hline Benzodiazepines & 879 & 4.089 \\
\hline Tricyclic antidepressants & 992 & 4.615 \\
\hline Selective serotonin reuptake inhibitors & 46 & 0.214 \\
\hline Lithium & 8 & 0.037 \\
\hline Psychotropics & 960 & 4.466 \\
\hline Carbamazepine & 380 & 1.768 \\
\hline Other antiepileptics & 230 & 1.07 \\
\hline Pregabalin & 240 & 1.11 \\
\hline Anticholinergics & 6 & 0.027 \\
\hline Cogentin & 19 & 0.088 \\
\hline Baclofen & 114 & 0.53 \\
\hline Analgesics & 1614 & 7.5 \\
\hline NSAIDs & 676 & 3.145 \\
\hline Acetaminophen-containing analgesics & 833 & 3.875 \\
\hline Salicylates & 105 & 0.488 \\
\hline Cardiovascular drugs & 1435 & 6.7 \\
\hline Theophylline & 611 & 2.842 \\
\hline Beta Blockers & 507 & 2.36 \\
\hline Antihypertensives & 287 & 1.33 \\
\hline Digoxin & 18 & 0.083 \\
\hline Calcium channel antagonists & 12 & 0.05 \\
\hline Antidiabetic medications & 475 & 2.2 \\
\hline Insulin and oral hypoglycemic drugs & 475 & 2.2 \\
\hline Others & 3706 & 17.2 \\
\hline Antibiotics & 511 & 2.377 \\
\hline Methotrexate & 12 & 0.05 \\
\hline Oral contraceptive pills & 183 & 0.851 \\
\hline Antihistaminics & 558 & 2.596 \\
\hline Vitamins & 328 & 1.526 \\
\hline Flagyl & 131 & 0.609 \\
\hline Miscellaneous & 446 & 2.07 \\
\hline Unknown & 1537 & 7.15 \\
\hline Total & 12797 & 59.5 \\
\hline
\end{tabular}

N: Number of patients, \%: Percentage, NSAIDs: Non-steroidal anti-inflammatory drugs 
Table (8): Non-drug most frequently involved in poisoned cases received in PCC-ASUH during the year 2019

\begin{tabular}{|c|c|c|}
\hline Type & $\mathbf{N}$ & $\%$ \\
\hline Food poisoning & 1649 & 7.7 \\
\hline \multirow{3}{*}{$\begin{array}{l}\text { Bacterial } \\
\text { Ciguatara } \\
\text { Botulism } \\
\end{array}$} & 1629 & 7.6 \\
\hline & 14 & 0.065 \\
\hline & 6 & 0.03 \\
\hline Insecticides \& rodenticides & 2644 & 12.29 \\
\hline \multirow{3}{*}{$\begin{array}{l}\text { Organophosphate insecticides } \\
\text { Zinc Phosphide } \\
\text { Aluminium phosphide }\end{array}$} & 1958 & 9.11 \\
\hline & 640 & 2.97 \\
\hline & 46 & 0.21 \\
\hline Household chemicals & 2891 & 13.4 \\
\hline \multirow{4}{*}{$\begin{array}{l}\text { Corrosives } \\
\text { Detergents } \\
\text { Kerosene and petroleum distillates } \\
\text { Phenol }\end{array}$} & 1394 & 6.49 \\
\hline & 918 & 4.27 \\
\hline & 479 & 2.23 \\
\hline & 100 & 0.46 \\
\hline Animal poisoning & 483 & 2.25 \\
\hline Scorpion & 334 & 1.55 \\
\hline Snake & 149 & 0.69 \\
\hline Toxic gases & 499 & 2.32 \\
\hline Carbon Monoxide & 494 & 2.3 \\
\hline Hydrogen sulfide & 5 & 0.02 \\
\hline Alcohols & 468 & 2.18 \\
\hline \multirow{2}{*}{$\begin{array}{l}\text { Ethanol } \\
\text { Methanol }\end{array}$} & 426 & 1.98 \\
\hline & 42 & 0.2 \\
\hline Metals & 12 & 0.056 \\
\hline Lead & 5 & 0.02 \\
\hline Mercury & 2 & 0.01 \\
\hline Cyanide & 5 & 0.02 \\
\hline Other non-drug agents & 49 & 0.23 \\
\hline \multirow{5}{*}{$\begin{array}{l}\text { Plants and herbals } \\
\text { Phenylenediamine (PPD) } \\
\text { Methemogobinemia } \\
\text { Nicotine } \\
\text { Dormex }\end{array}$} & 8 & 0.04 \\
\hline & 29 & 0.13 \\
\hline & 3 & 0.01 \\
\hline & 4 & 0.02 \\
\hline & 5 & 0.02 \\
\hline Total & 8695 & 40.5 \\
\hline
\end{tabular}

N: Number of patients, \%: Percentage

Table (9): Top ten most frequently involved exposure substances received in PCC-ASUH during the year 2019

\begin{tabular}{|c|c|c|}
\hline All exposures & $\mathbf{N}$ & $\mathbf{\%}$ \\
\hline Corrosives and detergents & 2312 & 10.76 \\
\hline Organophosphate insecticides & 1958 & 9.11 \\
\hline Food poisoning & 1649 & 7.7 \\
\hline Tricyclic antidepressants & 992 & 4.61 \\
\hline Psychotropics & 960 & 4.46 \\
\hline Cannabis & 936 & 4.35 \\
\hline Bcetaminophen-containing analgesics & 879 & 4.08 \\
\hline NSAIDs & 833 & 3.87 \\
\hline Zinc Phosphide & 676 & 3.14 \\
\hline
\end{tabular}

N: Number of patients, \%: Percentage, NSAIDs: Non-steroidal anti-inflammatory drugs 
Table (10): Severity grading of poisoning cases received in PCC-ASUH during the year 2019 according to poisoning severity score (Persson et al., 1998)

\begin{tabular}{|c|c|c|}
\hline PSS & N & \% \\
\hline Mild (1) & 17066 & 79.4 \\
\hline Moderate (2) & 3084 & 14.4 \\
\hline Severe (3) & 1342 & 6.2 \\
\hline Total & $\mathbf{2 1 4 9 2}$ & $\mathbf{1 0 0}$ \\
\hline
\end{tabular}

N: Number of patients, \%: Percentage, PSS: Poisoning severity score

Table (11): Period of hospitalization of poisoned cases received in PCC-ASUH during the year 2019

\begin{tabular}{|c|c|c|}
\hline Period of hospitalization & $\mathbf{N}$ & $\mathbf{\%}$ \\
\hline$<6$ hrs & 17066 & 79.4 \\
\hline 6 hrs -1 day & 3106 & 14.5 \\
\hline$>1$ day -4 days & 1160 & 5.4 \\
\hline$>4$ days -7 days & 88 & 0.4 \\
\hline$>7$ days & 72 & 0.3 \\
\hline Total & $\mathbf{2 1 4 9 2}$ & $\mathbf{1 0 0}$ \\
\hline
\end{tabular}

$N$ : Number of patients, \%: Percentage

Table (12): Outcome of acutely poisoned cases received in PCC-ASUH through year 2019

\begin{tabular}{|c|c|c|}
\hline Outcome & $\mathbf{N}$ & $\mathbf{\%}$ \\
\hline Discharge with complete improvement & 20885 & 97.2 \\
\hline Discharge against medical advice & 464 & 2.2 \\
\hline Death & 143 & 0.7 \\
\hline Total & $\mathbf{2 1 4 9 2}$ & $\mathbf{1 0 0}$ \\
\hline
\end{tabular}

N: Number of patients, \%: Percentage

Table (13): Death-related offending agents in 143 acutely poisoned cases received in PCC-ASUH through year 2019

\begin{tabular}{|l|c|c|l|c|c|}
\hline \multicolumn{1}{|c|}{ Agent } & N & \% & \multicolumn{1}{c|}{ Agent } & N & \% \\
\hline Organophosphates & 34 & 23.8 & Calcium channel antagonists & 3 & 2.1 \\
\hline Opiates & 21 & 14.7 & Theophylline & 2 & 1.4 \\
\hline Aluminium phosphide & 20 & 13.9 & Pregabalin & 2 & 1.4 \\
\hline Methanol & 9 & 6.3 & Insulin \& oral hypoglycemic drugs & 1 & 0.7 \\
\hline Tramadol & 5 & 3.5 & Digoxin & 1 & 0.7 \\
\hline Carbon monoxide & 5 & 3.5 & Paraphenylenediamine & 1 & 0.7 \\
\hline Corrosives & 5 & 3.5 & Methemoglobinemia & 1 & 0.7 \\
\hline Psychotropics & 4 & 2.8 & Dormex & 1 & 0.7 \\
\hline Zinc phosphide & 4 & 2.8 & Acetaminophen & 1 & 0.7 \\
\hline Carbamazepine & 3 & 2.1 & Cannabis & 0.7 \\
\hline Methotrexate & 3 & 2.1 & Hydrogen sulfide & 1 & 0.7 \\
\hline Benzodiazepines & 3 & 2.1 & Unknown & 12 & 8.4 \\
\hline Total & $\mathbf{1 4 3}$ & $\mathbf{0 . 6 6}$ & & & \\
\hline
\end{tabular}


Table (14): Emergency treatment and interventions offered to acutely poisoned cases received in PCC-ASUH during the year 2019

\begin{tabular}{|c|c|}
\hline Type of intervention & $\mathbf{N}(\%)$ \\
\hline Endotracheal intubation & $268(1.24)$ \\
\hline Mechanical Ventilation & $255(1.19)$ \\
\hline Dopamine & $58(0.27)$ \\
\hline \multicolumn{2}{|c|}{ Decontamination (2\%) } \\
\hline Gastric lavage & $344(1.6)$ \\
\hline Skin wash & $88(0.4)$ \\
\hline \multicolumn{2}{|c|}{ Enhanced elimination (42.7\%) } \\
\hline Activated charcoal & $9102(42.3)$ \\
\hline Hemodialysis & $22(0.1)$ \\
\hline Sodium bicarbonate & $54(0.25)$ \\
\hline \multicolumn{2}{|c|}{ Antidotes (9.65\%) } \\
\hline Atropine & 1117 (5.19) \\
\hline Obidoxime & $475(2.2)$ \\
\hline Naloxone & $104(0.48)$ \\
\hline Scorpion anti-venom & $99(0.46)$ \\
\hline Snake anti-venom & $87(0.4)$ \\
\hline $\mathrm{N}$-acetylcysteine & $67(0.3)$ \\
\hline Hyperbaric O2 therapy & $35(0.16)$ \\
\hline Ethanol & $29(0.13)$ \\
\hline Flumazenil & $25(0.11)$ \\
\hline Folinic acid & $12(0.05)$ \\
\hline Glucagon & $11(0.05)$ \\
\hline Antibotulinum & $6(0.03)$ \\
\hline Calcium gluconate & $5(0.02)$ \\
\hline Methylene blue & $3(0.01)$ \\
\hline Total & 2075 \\
\hline
\end{tabular}

N: Number of patients \%: Percentage

Table (15): Chi- Square statistical analysis of types of toxic agents in acutely poisoned cases received in PCCASUH during 2019 regarding age groups

\begin{tabular}{|c|c|c|c|c|c|c|c|c|c|c|c|c|c|}
\hline \multirow{3}{*}{ Types of toxic agents } & \multicolumn{10}{|c|}{ Age (years) } & \multirow{3}{*}{$\begin{array}{c}\text { Total } \\
(\mathrm{N}= \\
21492)\end{array}$} & \multirow{3}{*}{$\mathrm{X}^{2}$} & \multirow{3}{*}{$\begin{array}{c}\text { P- } \\
\text { value }\end{array}$} \\
\hline & \multicolumn{2}{|c|}{$\begin{array}{c}<7 \\
(\mathrm{~N}=5666)\end{array}$} & \multicolumn{2}{|c|}{$\begin{array}{c}7-<15 \\
(N= \\
1269)\end{array}$} & \multicolumn{2}{|c|}{$\begin{array}{c}15-<25 \\
(N=7289)\end{array}$} & \multicolumn{2}{|c|}{$\begin{array}{c}25-40 \\
(N=5452)\end{array}$} & \multicolumn{2}{|c|}{$\begin{array}{c}>40 \\
(N= \\
1816)\end{array}$} & & & \\
\hline & $\mathbf{N}$ & $\%$ & $\mathbf{N}$ & $\%$ & $\mathbf{N}$ & $\%$ & $\mathbf{N}$ & $\%$ & $\mathrm{~N}$ & $\%$ & & & \\
\hline Therapeutic drugs & 1928 & 34 & 308 & 24.2 & 4437 & 60.9 & 3527 & 64.7 & 904 & 49.8 & 11104 & 1716.8 & $0.000 *$ \\
\hline $\begin{array}{c}\text { Drugs/substances } \\
\text { of abuse }\end{array}$ & 330 & 5.8 & 117 & 9.2 & 636 & 8.7 & 567 & 10.4 & 43 & 2.4 & 1693 & 219.9 & $0.000 *$ \\
\hline Insecticides/rodenticides & 709 & 12.5 & 355 & 27.9 & 855 & 11.7 & 570 & 10.5 & 155 & 8.5 & 2644 & 209.9 & $0.000 *$ \\
\hline Household chemicals & 2035 & 35.9 & 214 & 16.9 & 233 & 3.2 & 203 & 3.7 & 206 & 11.3 & 2891 & 3149.9 & $0.000^{*}$ \\
\hline Food poisoning & 397 & 7.1 & 203 & 16 & 487 & 6.7 & 289 & 5.3 & 273 & 15 & 1649 & 319.9 & $0.000 *$ \\
\hline Animal poisoning & 47 & 0.8 & 33 & 2.6 & 213 & 2.9 & 117 & 2.1 & 73 & 4 & 483 & 93.9 & $0.000 *$ \\
\hline Toxic gases & 90 & 1.6 & 15 & 1.2 & 190 & 2.6 & 104 & 1.9 & 100 & 5.5 & 499 & 196.9 & $0.000 *$ \\
\hline Alcohols & 125 & 2.2 & 18 & 1.4 & 220 & 3 & 55 & 1 & 50 & 2.8 & 468 & 65.4 & $0.000 *$ \\
\hline Others $^{\#}$ & 5 & 0.1 & 6 & 0.5 & 18 & 0.2 & 20 & 0.4 & 12 & 0.7 & 61 & 20.05 & $0.000 *$ \\
\hline
\end{tabular}

(\#): Metals and other non-drug agents $\quad\left(^{*}\right)$ : statistically significant difference 
Table (16): Chi- Square statistical analysis of types of toxic agents in acutely poisoned cases received in PCCASUH during 2019 regarding sex

\begin{tabular}{|c|c|c|c|c|c|c|c|}
\hline \multirow{3}{*}{ Types of toxic agents } & \multicolumn{4}{|c|}{ Sex } & \multirow{3}{*}{$\begin{array}{c}\text { Total } \\
(\mathrm{N}= \\
21492)\end{array}$} & \multirow{3}{*}{$X^{2}$} & \multirow{3}{*}{ P-value } \\
\hline & \multicolumn{2}{|c|}{ Males (N = 9721) } & \multicolumn{2}{|c|}{ Females $(N=11771)$} & & & \\
\hline & $\mathbf{N}$ & $\%$ & $\mathbf{N}$ & $\%$ & & & \\
\hline Therapeutic drugs & 4356 & 44.8 & 6748 & 57.3 & 11104 & 334.04 & $0.000^{*}$ \\
\hline Drugs/substances of abuse & 1180 & 12.1 & 513 & 4.4 & 1693 & 444.14 & $0.000^{*}$ \\
\hline Insecticides/rodenticides & 1131 & 11.6 & 1513 & 12.9 & 2644 & 7.33 & $0.007^{*}$ \\
\hline Household chemicals & 994 & 10.2 & 1897 & 16.1 & 2891 & 158.68 & $0.000^{*}$ \\
\hline Food poisoning & 1094 & 11.3 & 555 & 4.7 & 1649 & 321.36 & $0.000^{*}$ \\
\hline Animal poisoning & 349 & 3.6 & 134 & 1.1 & 483 & 145.68 & $0.000^{*}$ \\
\hline Toxic gases & 265 & 2.7 & 234 & 2 & 499 & 12.79 & $0.000^{*}$ \\
\hline Alcohols & 325 & 3.3 & 143 & 1.2 & 468 & 113.23 & $0.000^{*}$ \\
\hline Others $^{\#}$ & 27 & 0.3 & 34 & 0.3 & 61 & 0.023 & 0.879 \\
\hline
\end{tabular}

(\#): Metals and other non-drug agents, (*): statistically significant difference, P-value > 0.05: Non significant difference

Table (17): Age group distribution in relation to poisoning severity score, period of hospitalization and outcome in acutely poisoned cases received in PCC-ASUH during the year 2019

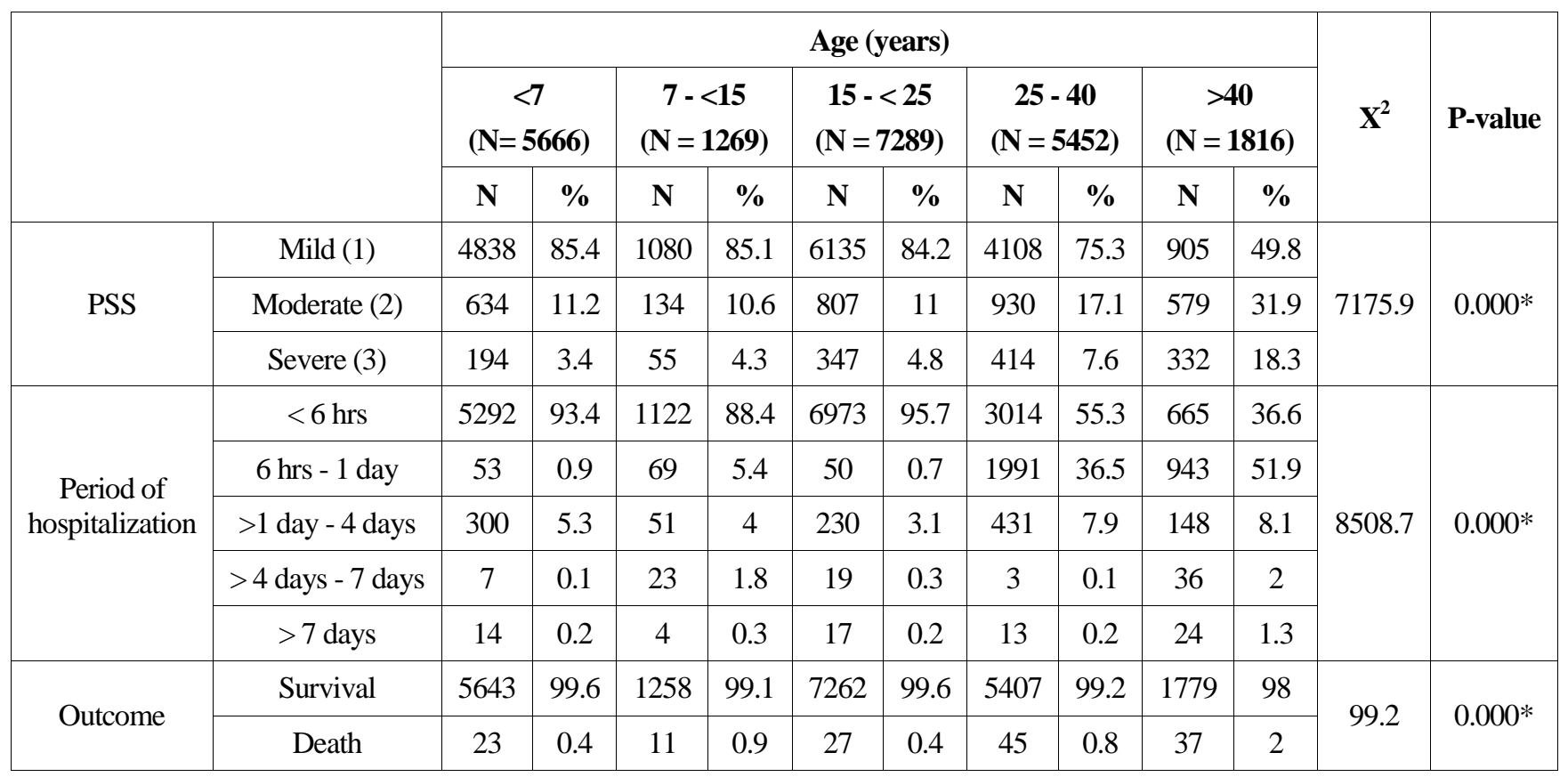

Chi square test was used, PSS: Poisoning severity score, $(*)$ : statistically significant difference 
Table (18): Sex distribution in relation to poisoning severity score, period of hospitalization and outcome in acutely poisoned cases received in PCC-ASUH during the year 2019

\begin{tabular}{|c|c|c|c|c|c|c|c|}
\hline & & \multicolumn{4}{|c|}{ Sex } & \multirow{3}{*}{$\mathbf{X}^{2}$} & \multirow{3}{*}{ P-value } \\
\hline & & \multicolumn{2}{|c|}{ Males (N = 9721) } & \multicolumn{2}{|c|}{ Females $(\mathrm{N}=11771)$} & & \\
\hline & & $\mathbf{N}$ & $\%$ & $\mathbf{N}$ & $\%$ & & \\
\hline \multirow{3}{*}{ PSS } & Mild (1) & 7682 & 79 & 9384 & 79.7 & \multirow{3}{*}{2.652} & \multirow{3}{*}{0.27} \\
\hline & Moderate (2) & 1405 & 14.5 & 1679 & 14.3 & & \\
\hline & Severe (3) & 634 & 6.5 & 708 & 6 & & \\
\hline \multirow{5}{*}{ Period of hospitalization } & $<6 \mathrm{hrs}$ & 7700 & 79.2 & 9366 & 79.6 & \multirow{5}{*}{8.036} & \multirow{5}{*}{0.09} \\
\hline & 6 hrs - 1 day & 1387 & 14.3 & 1719 & 14.6 & & \\
\hline & $>1$ day - 4 days & 565 & 5.8 & 595 & 5.1 & & \\
\hline & $>4$ days -7 days & 34 & 0.3 & 54 & 0.5 & & \\
\hline & $>7$ days & 35 & 0.4 & 37 & 0.3 & & \\
\hline \multirow{2}{*}{ Outcome } & Survival & 9651 & 99.3 & 11698 & 99.4 & \multirow{2}{*}{0.804} & \multirow{2}{*}{0.37} \\
\hline & Death & 70 & 0.7 & 73 & 0.6 & & \\
\hline
\end{tabular}

Chi square test was used, PSS: Poisoning severity score, P-value > 0.05: Non significant difference

Table (19): Poisoning severity score in relation to period of hospitalization and outcome in acutely poisoned cases received in PCC-ASUH during the year 2019

\begin{tabular}{|c|c|c|c|c|c|c|c|c|c|}
\hline & \multicolumn{6}{|c|}{ PSS } & \multirow{3}{*}{$X^{2}$} & \multirow{3}{*}{ P-value } \\
\hline & & \multicolumn{2}{|c|}{ Mild (1) (N= 17066) } & \multicolumn{2}{|c|}{ Moderate (2) $(\mathrm{N}=3084)$} & \multicolumn{2}{|c|}{ Severe (3) $(N=1342)$} & & \\
\hline & & $\mathbf{N}$ & $\%$ & $\mathbf{N}$ & $\%$ & $\mathbf{N}$ & $\%$ & & \\
\hline \multirow{5}{*}{$\begin{array}{c}\text { Period of } \\
\text { hospitalization }\end{array}$} & $<6$ hrs & 15816 & 92.7 & 1244 & 40.3 & 6 & 0.4 & \multirow{5}{*}{22833.65} & \multirow{5}{*}{$0.000^{*}$} \\
\hline & $\begin{array}{c}6 \text { hrs } \\
-1 \text { day }\end{array}$ & 1241 & 7.3 & 1736 & 56.3 & 129 & 9.6 & & \\
\hline & $\begin{array}{l}>1 \text { day } \\
-4 \text { days }\end{array}$ & 9 & 0.1 & 79 & 2.6 & 1072 & 79.9 & & \\
\hline & $\begin{array}{l}>4 \text { days } \\
-7 \text { days }\end{array}$ & 0 & 0.0 & 10 & 0.3 & 78 & 5.8 & & \\
\hline & $>7$ days & 0 & 0.0 & 15 & 0.5 & 57 & 4.2 & & \\
\hline \multirow{2}{*}{ Outcome } & Survival & 17063 & 99.9 & 3049 & 98.8 & 1237 & 92.2 & \multirow{2}{*}{1166.2} & \multirow{2}{*}{$0.000^{*}$} \\
\hline & Death & 3 & 0.01 & 35 & 1.1 & 105 & 7.8 & & \\
\hline
\end{tabular}

Chi square test was used, PSS: Poisoning severity score, $(*)$ : statistically significant difference

Table (20): Relation between outcome and period of hospitalization in acutely poisoned cases received in PCCASUH during the year 2019

\begin{tabular}{|c|c|c|c|c|}
\hline \multirow{3}{*}{$\begin{array}{c}\text { Period of } \\
\text { hospitalization }\end{array}$} & \multicolumn{2}{|c|}{ Outcome } & \multirow{3}{*}{$X^{2}$} & \multirow{3}{*}{ P-value } \\
\hline & Survivors $(N=21349)$ & Non-survivors & & \\
\hline & N (\%) & No. $=143$ & & \\
\hline$<6$ hrs & 17057 (79.9\%) & $9(6.3 \%)$ & \multirow{5}{*}{2155.4} & \multirow{5}{*}{$0.000^{*}$} \\
\hline 6 hrs - 1 day & 3087 (14.5\%) & 19 (13.3\%) & & \\
\hline$>1$ day - 4 days & 1085 (5.1\%) & 75 (52.4\%) & & \\
\hline$>4$ days - 7 days & $69(0.3 \%)$ & 19 (13.3\%) & & \\
\hline$>7$ days & $51(0.2 \%)$ & $21(14.7 \%)$ & & \\
\hline
\end{tabular}

Chi square test was used, (*): statistically significant difference 
Table (21): Relation between types of toxic agents and outcome in acutely poisoned cases received in PCC-ASUH during the year 2019

\begin{tabular}{|c|c|c|c|c|}
\hline \multirow{2}{*}{ Toxic agents } & Survivors $(\mathrm{N}=11993)$ & Non survivors $(N=143)$ & \multirow{2}{*}{$X^{2}$} & \multirow{2}{*}{ P-value } \\
\hline & $\mathbf{N}(\%)$ & $\mathbf{N}(\%)$ & & \\
\hline Organophosphates & $1924(16 \%)$ & $34(23.8 \%)$ & 6.246 & $0.012^{*}$ \\
\hline Opiates & $361(3 \%)$ & $21(14.7 \%)$ & 63.186 & $0.000^{*}$ \\
\hline Aluminium phosphide & $26(0.2 \%)$ & $20(13.9 \%)$ & 709.535 & $0.000^{*}$ \\
\hline Methanol & $33(0.3 \%)$ & $9(6.3 \%)$ & 148.424 & $0.000^{*}$ \\
\hline Tramadol & $240(2 \%)$ & $5(3.5 \%)$ & 1.597 & 0.206 \\
\hline Carbon monoxide & $489(4.1 \%)$ & $5(3.5 \%)$ & 0.122 & 0.727 \\
\hline Corrosives & $1389(11.6 \%)$ & $5(3.5 \%)$ & 9.086 & $0.003^{*}$ \\
\hline Psychotropics & $956(8 \%)$ & $4(2.8 \%)$ & 5.193 & $0.023^{*}$ \\
\hline Zinc phosphide & $636(5.3 \%)$ & $4(2.8 \%)$ & 1.776 & 0.183 \\
\hline Carbamazepine & $377(3.1 \%)$ & $3(2.1 \%)$ & 0.509 & 0.476 \\
\hline Methotrexate & $9(0.1 \%)$ & $3(2.1 \%)$ & 58.539 & $0.000^{*}$ \\
\hline Benzodiazepines & $876(7.3 \%)$ & $3(2.1 \%)$ & 5.702 & $0.017^{*}$ \\
\hline Calcium channel antagonists & $9(0.1 \%)$ & $3(2.1 \%)$ & 58.539 & $0.000^{*}$ \\
\hline Theophylline & $609(5.1 \%)$ & $2(1.4 \%)$ & 4.001 & $0.045^{*}$ \\
\hline Pregabalin & $238(2 \%)$ & $2(1.4 \%)$ & 0.250 & 0.617 \\
\hline Insulin and oral hypoglycemic drugs & $474(4 \%)$ & $1(0.7 \%)$ & 3.976 & $0.046^{*}$ \\
\hline Digoxin & $17(0.1 \%)$ & $1(0.7 \%)$ & 2.966 & 0.085 \\
\hline Paraphenylenediamine & $28(0.2 \%)$ & $1(0.7 \%)$ & 1.286 & 0.257 \\
\hline Methemoglobinemia & $2(0.02 \%)$ & $1(0.7 \%)$ & 26.645 & $0.000^{*}$ \\
\hline Dormex & $4(0.03 \%)$ & $1(0.7 \%)$ & 15.218 & $0.000^{*}$ \\
\hline Acetaminophen & $832(6.9 \%)$ & $1(0.7 \%)$ & 8.602 & $0.003^{*}$ \\
\hline Cannabis & $935(7.8 \%)$ & $1(0.7 \%)$ & 10.000 & $0.002^{*}$ \\
\hline Hydrogen sulfide & $4(0.03 \%)$ & $1(0.7 \%)$ & 15.218 & $0.000^{*}$ \\
\hline Unknown & $1525(12.7 \%)$ & $12(8.4 \%)$ & 2.389 & 0.122 \\
\hline
\end{tabular}

Chi square test was used, (*): statistically significant difference, $P$-value $>0.05$ : Non significant difference

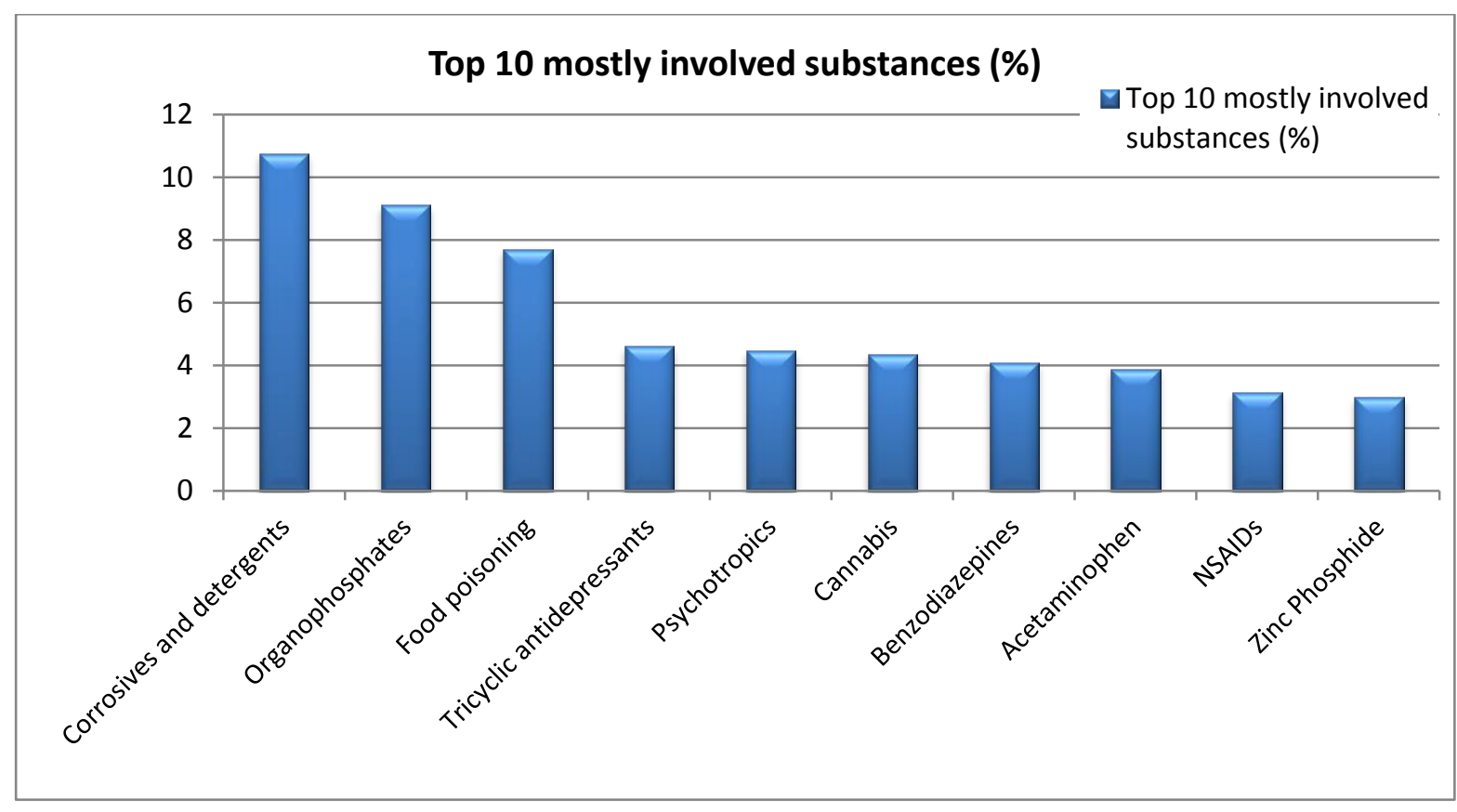

Figure (1): Top 10 most frequently involved substances in PCC-ASUH during the year 2019. 


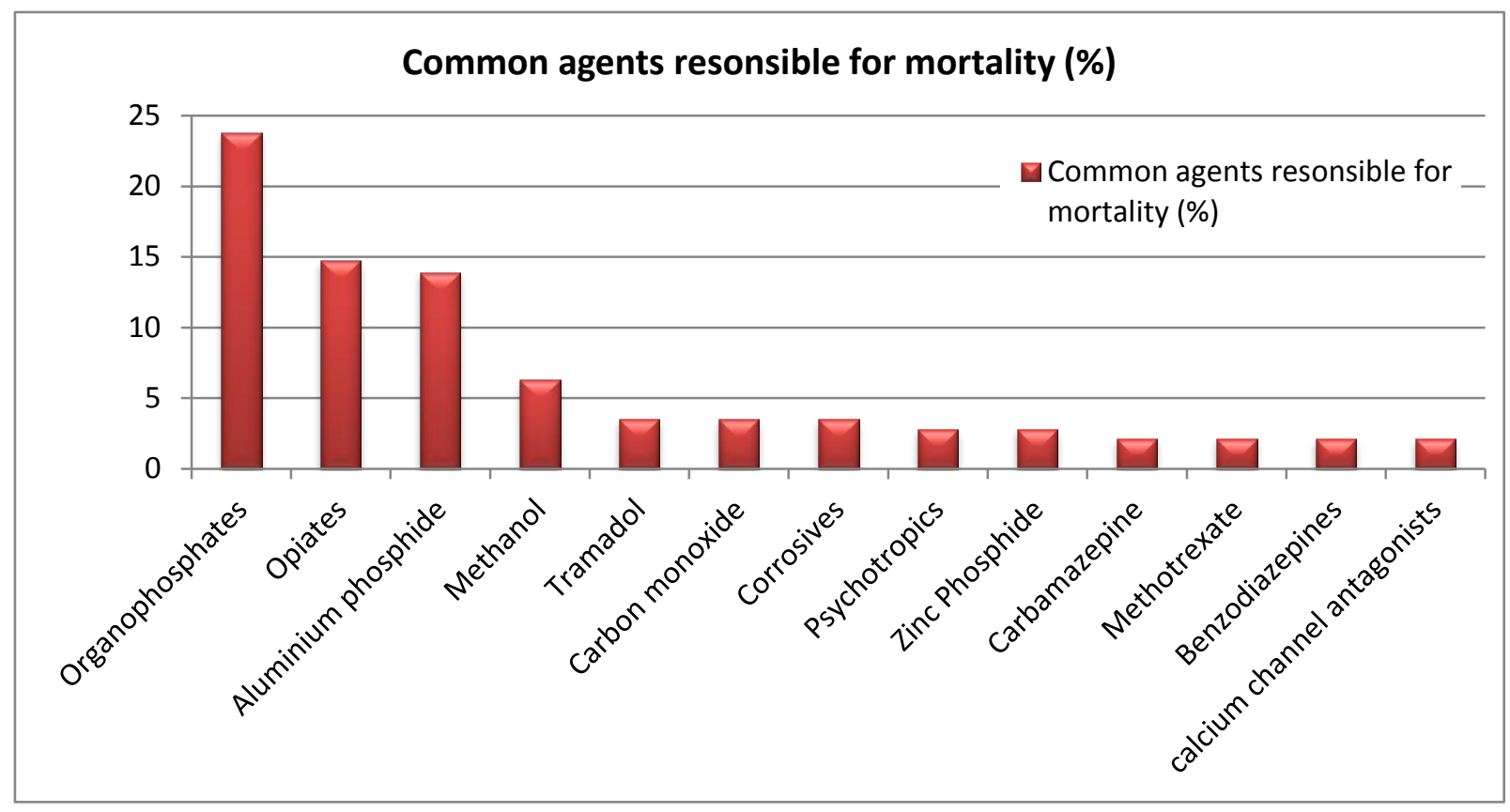

Figure (2): Common agents responsible for mortality in PCC-ASUH during the year 2019.

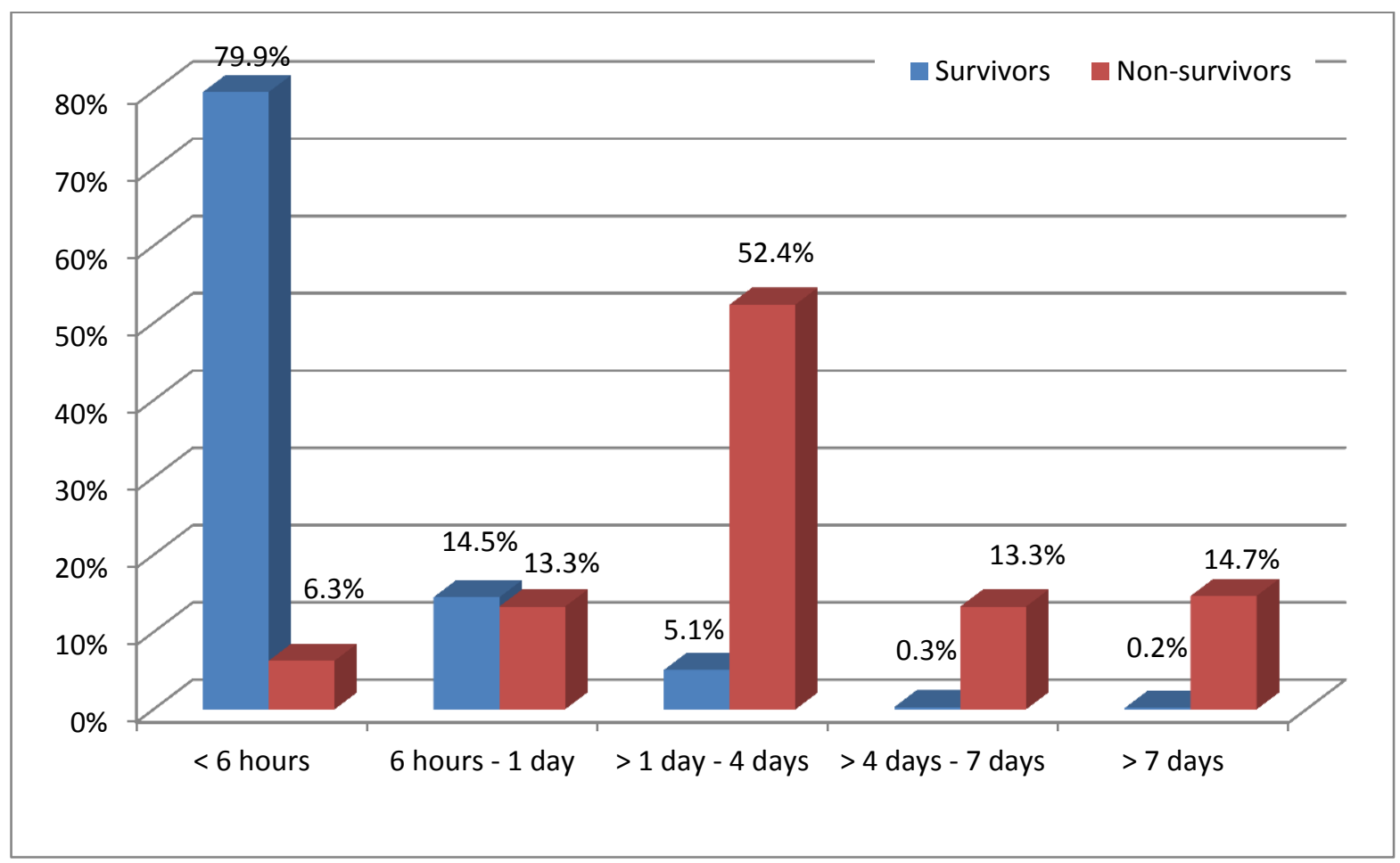

Figure (3): Relation between the outcome and hospital stay in the studied cases. 


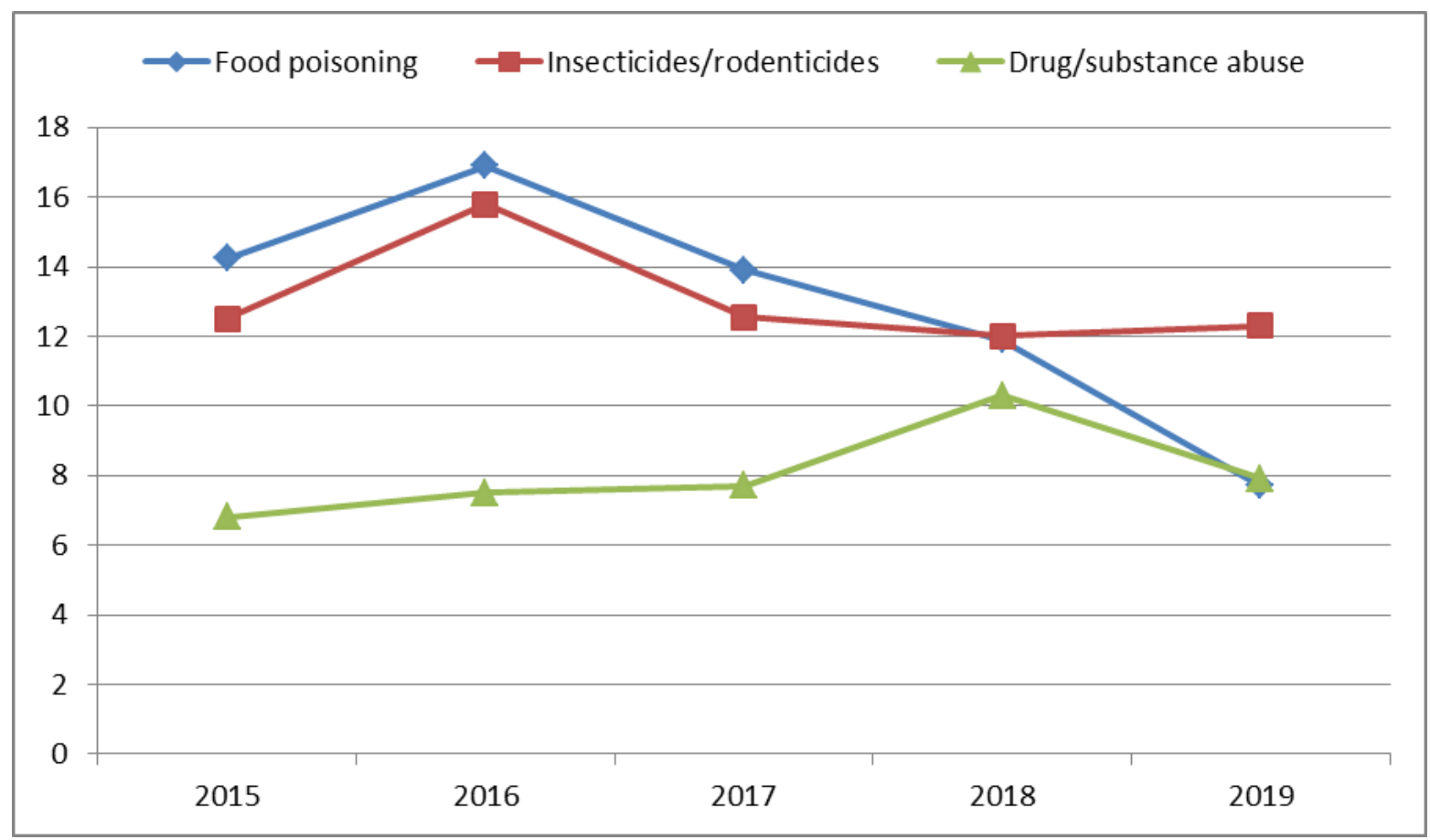

Figure (4): Yearly variation of some types of intoxication presented to PCC-ASUH.

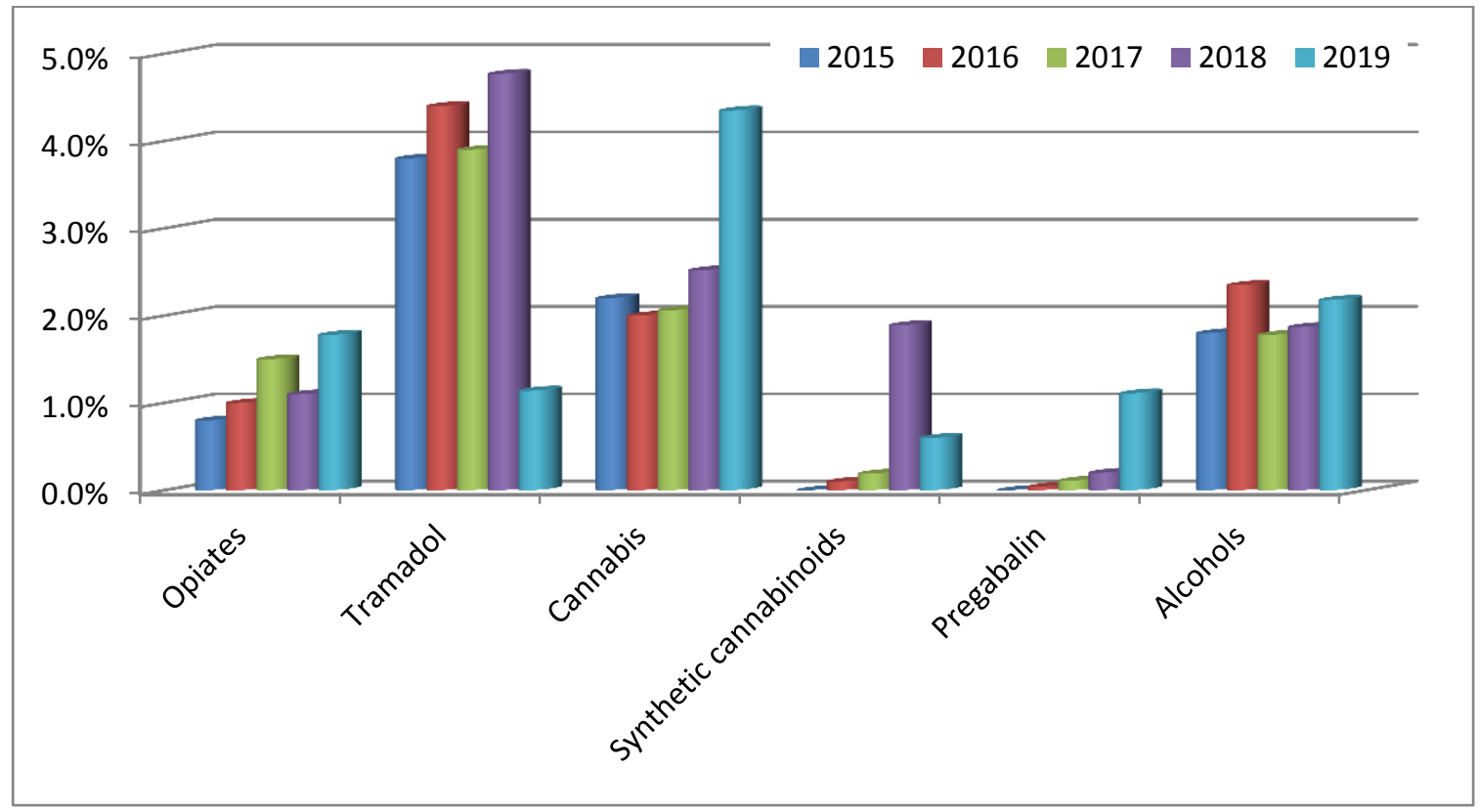

Figure (5): Yearly variation of drug/substance abuse presented to PCC-ASUH.

\section{Discussion}

Toxicological exposures, whether intentional or unintentional, continue to be a significant cause of morbidity and mortality in Egypt. Poison Control Center of Ain Shams University hospitals is the first and largest national poison treatment center in Egypt where thousands of poisoned patients are received yearly. The current study included a total number of 21,492 poisoned patients presented to the PCC-ASUH during the year 2019 .

The study revealed that the majority of cases $(59.3 \%)$ were from 15 to 40 years old followed by the age group less than seven years $(26.4 \%)$. This came in accordance with previous PCC-ASUH annual reports where poisoned patients between 15 and 40 years ranged between 59.2\% and 63.5\% (Halawa et al., 2013; Tawfik and ElHelaly, 2015; Tawfik and Khalifa 2017). Additionally, other studies observed that acute poisoning was prevalent among younger adults with less than $3 \%$ of toxic exposures observed in people aged 60 years or older (Cassidy et al., 2008; Karbakhsh and Zandi, 2008). The high incidence of poisoning in this age group deserves particular attention and could be attributed to 
tremendous emotional, social, political and economic challenges in Egypt (El Masry and Tawfik, 2013). In contrast, a similar Egyptian study done by Hegazy and Elfiky (2016) in Menoufia showed that poisoned cases aged less than seven years predominated (40.1\%) followed by those aged from seven to 15 years $(27.9 \%)$. In addition, the 2018 annual report of the American Association of Poison Control Centers' National Poison Data System (NPDS) revealed that approximately half of poisonings $(44.2 \%)$ occurred in children aged five years and younger (Gummin et al., 2019).

In the current study, $54.8 \%$ of poisoned patients were females and $45.2 \%$ were males. This was in agreement with an Egyptian study done by $\mathrm{Abo} \mathrm{El-}$ Noor (2013) and an Ethiopian study done by Adinew and Asrie (2016) where females represented 57.1\% and $63.5 \%$ of their patients respectively. On the contrary, these results disagreed with those obtained by Anthony and Kulkarni (2012) and Prayag et al. (2016) who reported that male poisoned patients outnumbered females in India, representing $52.5 \%$ and $62.7 \%$ of cases respectively. Female preponderance in our study could be due to higher rate of psychological stress, family conflicts and emotional liability in young women with their tendency to prefer suicidal attempts using different poisons or drugs as a way of salvation.

The majority of cases in this study originated from Cairo (66.5\%) which was largely expected as the PCC-ASUH is located in Cairo. This distribution was similar to data recorded during the year 2015 which revealed that most of cases came from Cairo (69.2\%) (Tawfik and Khalifa, 2017). Poisoned patients in other governorates could have been treated in their regional poison control centers or district hospitals.

More than $89 \%$ of studied poisoned cases were exposed through oral route while other routes represented only $10.9 \%$. This agreed with Hegazy and Elfiky (2016) and Asawari et al. (2017) where ingestion was the main route of exposure. Oral route could reflect the increased potential for suicidal attempts or household exposure and decreased possibility of occupational and environmental toxicities in our study.

Despite the strong religious morals that ban selfdestruction and deliberate self-killing, yet suicidal attempts occurred in $51.5 \%$ of our studied patients followed by accidental poisoning (39.6\%). These results were in accordance with Adinew and Asrie (2016) and Bamathy et al. (2017). Acute deliberate self-poisoning is mostly a dynamic medical illness that represents acute exacerbation of a chronic underlying psychological disorder (Moghaddam et al., 2014). Suicidal attempts could be also linked to several social reasons as poverty, unemployment, stress owing to family burden, serious health problems, substance abuse, educational issues (e.g. failure in examinations or lack of financial support), and disappointment in love affairs (Prayag et al., 2016). In contrast, the 2018 annual report of the American Association of Poison Control Centers found that only $19.1 \%$ of poisoning was intentional (Gummin et al., 2019).

The current study declared that drug poisoning was more prevalent cause of poisoning (59.5\%) compared to non-drug poisoning $(40.5 \%)$, bearing a great resemblance to the data issued by Wijegoonawardene et al. (2015) and Hegazy and Elfiky (2016) where drugs were responsible for poisoning in $63.6 \%$ and $52.9 \%$ of their studied patients, respectively. On the other hand, Sigdel et al. (2019) recorded non-drug poisoning, mainly by organophosphorous insecticides in $68.4 \%$ of their poisoned patients in Nepal.

It was obvious that centrally acting drugs constituted $18 \%$ of toxic exposures in the current study, where antidepressants predominated $(4.6 \%)$ followed by psychotropics $(4.46 \%)$. This was in agreement with poisoning pattern of cases presented to PCC-ASUH during 2015 (Tawfik and Khalifa, 2017) and Bamathy et al. (2017). This could be attributed to the availability of medications among the general population without restriction rules or banning programs enrolled by the government. In contrast, Asawari et al. (2017) reported NSAIDs and analgesics as the major causes of drug poisoning in their study.

Drug of abuse poisoning constituted $7.9 \%$ of presentations in 2019 compared to $10.3 \%$ in 2018 (as shown in figure 4) and cannabis represented the majority of cases $(4.35 \%)$ followed by opiates $(1.78 \%)$. Though an alarming figure has been observed following the progressive rise in synthetic cannabinoids exposures recently, reaching 472 cases in 2018 (1.89\% of all cases), a sharp decrease was found in our study where they accounted for 130 cases $(0.6 \%)$ (PCC-ASUH annual report, 2018). The recent emergence of synthetic cannabinoids is mainly connected to their ability to induce psychoactive effects but with negative drug screening test. In addition, they are highly available with easy access and have lower costs compared to traditional cannabis (Zanda and Fattore, 2018). The observed reduction in synthetic cannabinoids exposures during 2019 was most likely attributed to their placement into Schedule I and/or reduced use due to increased public awareness regarding their toxicity (Hassen et al., 2018). Another observed result is the continuous increase in pregabalin exposures in the present study, rising from only 9 cases $(0.04 \%)$ reported during 2016 to 240 cases (1.11\%) during 2019 (PCC-ASUH annual report, 2016). Recently, pregabalin is used by opiate and benzodiazepine abusers to potentiate the effect of their abused drugs or as a substitute for their high abuse potential. Potent binding of pregabalin at calcium channels results in reduction in the release of excitatory molecules. Furthermore, it is thought to possess GABA-mimetic properties presented with direct and indirect effects on the dopaminergic 'reward' system (Schifano, 2014). Despite low pregabalin addictive liability at therapeutic dosages, misusers' perceptions for these molecules to constitute a valid substitute for common illicit drugs may be a reason of concern. These yearly variations (shown in figure 5) enable the PCCASUH to declare new recommendations concerning drug of abuse screening lists and issue several warnings in media that are given much concern by health and drugs of abuse authorities.

The results from our research revealed that the top five mostly involved toxic substances were corrosives and detergents $(10.76 \%)$, organophosphates 
$(9.11 \%)$, food poisoning $(7.7 \%)$, tricyclic antidepressants $(4.61 \%)$ and finally psychotropics $(4.46 \%)$. Household products are easily available with high concentration ingredients without safety manufacturing measures. They are often stored in an improper manner due to lack of facilities and community awareness of their hazards. This agreed with the 2015 annual report of the Australian Poisons Information Centers where household cleaning substances topped their list of toxic substances $(14.5 \%)$ followed by acetaminophencontaining analgesics (7.3\%) then antidepressants (4.2\%) (Huynh et al., 2018). On the contrary, drug poisoning made up the largest proportion of poisoning exposures $(43.5 \%)$ followed by pesticides $(37.5 \%)$ in a similar Indian study implemented by Nair and Revi (2015). In addition, Sande and kumar (2017) stated that snake bites exceeded $30 \%$ in their Indian studied patients. This affirms variations in toxicological profiles in different countries and sometimes within different regions in the same country.

As regards types of toxic agents in relation to age in this study, there was statistically significant differences as household chemicals were more prevalent in poisoned cases $<7$ years $(35.9 \%)$ followed by therapeutic drugs $(34 \%)$. This can be attributed to the specific behavior of this age group characterized by curiosity, oral identification, inability to discriminate in addition to taste and smell immaturity with availability and easy access to household agents. Insecticides were the most prevalent type in the age group from $7-<15$ years $(27.9 \%)$. This agreed with Azab et al. (2015) who found that pesticides were the commonest nonpharmaceutical agents in adolescents. On the other hand, drug poisoning was more prevalent in the age groups from $15-<25$ years $(60.9 \%), 25-40$ years $(64.7 \%)$ and $>40$ years $(49.8 \%)$. Similar results were reported by Hegazy and Elfiky (2016).

The current study showed that poisoning by substance abuse was significantly common in males compared to females $(12.1 \%$ versus $4.4 \%)$. This was similar to Abo El-Noor (2013). Foto-Özdemir et al. (2016) also stated that self-poisoning by drugs is mostly the main manner of intoxication in females, whereas drug abuse and addiction is more common in males.

This study showed that poisoning severity was mild in the majority $(79.4 \%)$ of cases, moderate in $(14.4 \%)$ and severe in (6.2\%). Similar results were found by Tawfik and ElHelaly (2015). Poisoning severity score is a simple scoring system that can be used as a mode of grading of poisoning in peripheral hospitals, which subsequently helps in timely referral of patients. On the other hand, Prayag et al. (2016) recorded PSS (1) in $42.7 \%$ of their studied patients, PSS (2) in $35.4 \%$ and documented severe intoxication in $21.8 \%$ of patients.

The recorded mortality rate in our study was $0.66 \%$ which agreed with Tawfik and Khalifa (2017) who recorded a mortality rate of $0.56 \%$ in their study. A mortality rate exceeding $25 \%$ was reported by Prayag et al. (2016) attributing variations in mortality rates to several factors that contribute in deciding the fate of the patient as the type of poison exposures; time elapsed from exposure to arrival to poison control center; proper assessment of severity of intoxication; availability of life-saving measures and transport services; and provision of spot laboratory diagnosis.

The present study showed that increasing age was associated with higher PSS, prolonged hospital stay and higher mortality rate. Nearly similar results were obtained by Barratt et al. (2013). Additionally, higher PSS was significantly associated with prolonged hospital stay and death. This was in agreement with Yuan et al. (2018) who observed an association between severe clinical course of poisoning and death.

The most frequent cause of death was organophosphates in our study representing $23.8 \%$ of total number of deaths. They used to be the first cause of poisoning-related deaths in Egypt for several years as documented by the 2011-2015 PCC-ASUH annual reports. Wijegoonawardene et al. (2015) stated that organophosphates had also the first rank of deaths due to poisoning in Sri Lanka (42.9\%). The high percentage of organophosphate poisoned cases, severity of manifestations and necessity for ICU admission with relatively high frequency of mechanical ventilation and the large number of fatalities make organophosphates poisoning of particular importance to health care system and invite for more rigid regulations concerning their misuse. In contrast, Hegazy and Elfiky (2016) reported death due to poisoning with aluminum and zinc phosphide in $60 \%$ of their deceased patients followed by organophosphates while fentanyl was the first cause of death in United States followed by acetaminophen (Gummin et al., 2019). Despite being the second cause of poisoning-induced mortality during the years from 2011 to 2017 , tramadol is now responsible for about $1.14 \%$ of the toxicities and only $3.5 \%$ of the deaths. This may be due to government stringency to punish those who sell, buy, or consume various drugs of abuse. However, development of its severe toxic effects mandates patients' referral to poison control centers despite fear government prosecution.

On studying the relation between different toxic agents responsible for mortality and outcome, a significant difference was found between survivors and non-survivors regarding several toxins (e.g. organophosphates, opiates, aluminium phosphide, methanol, corrosives and psychotropics) while no significant difference was observed with other toxins (e.g. tramadol, carbon monoxide, zinc phosphide and carbamazepine). Despite the importance of identifying toxic agents significantly associated with death, poisoning-related mortality varies widely and is influenced by many factors like increasing age, delay time, compromised immunity and presence of multiple comorbidities (Arun et al., 2005).

As regards hospital period of stay, this study declared that most of the cases $(79.4 \%)$ were observed for a period not exceeding six hours and $14.5 \%$ stayed for six hours to one day while $5.8 \%$ were admitted for one to seven days. Only $0.3 \%$ of patients were admitted for a period exceeding seven days. This agreed with Tawfik and ElHelaly (2015). Jalali et. al. (2012) also reported that $82.8 \%$ of their cases did not need admission. Mild severity of intoxication seen in most 
of our patients could explain this small admission rate. In a study done by Adinew and Asrie (2016), only 48\% of patients were discharged from the ER. Longer hospital stay was explained by Anthony and Kulkarni (2012) who postulated that duration of hospital stay and ventilator requirement depend on the nature and quantity of poison, presence of medical co-morbidities and poison-related complications. Moreover, the duration of hospitalization correlated significantly with mortality in this study which agreed with Tawfik and Khalifa (2017). In contrast, Anthony and Kulkarni (2012) reported that duration of hospital stay did not correlate with mortality.

In the present study, activated charcoal was the most frequent procedure undertaken in $42.3 \%$ of poisoned cases. The most frequently used antidotes were atropine and obidoxime reflecting the large number of received organophosphate poisoned cases.

\section{Conclusion}

This study demonstrated the magnitude of poisoning in a particular region of Egypt. It revealed that young and middle aged people were the most vulnerable for acute poisoning episodes. In addition, children younger than seven years accounted for more than $26 \%$ of cases. Females outnumbered males. Ingestion was the main route of poisoning and suicidal poisoning predominated. Corrosives and detergents were the most commonly involved toxic substances followed by organophosphates while antidepressants and psychotropics topped the list of drug poisoning. Poisoning severity score was mild in the majority of patients. Higher fatality rate for certain poisons compared to others could assist emergency physicians to triage poisoning cases in an overcrowded emergency department.

\section{Recommendations}

As presented by our study, PSS is a simple tool that could be involved in the training module of medical officers for better assessment and referral. A new governmental policy is needed to control medication marketing with strict regulations for household products and insecticides free availability. Legislations should be implemented to sell potentially dangerous chemicals in childproof containers and to ban the sale of commonly abused drugs without proper prescription by a qualified specialist. Collaboration between poison centers all over the country is necessary to provide a nation-wide surveillance for accurate mapping of poisoning in Egypt and to outline a unified protocol. Patients with suicidal poisoning should undergo compulsory in-patient psychiatric consultation to reduce the risk of future attempts. Provision of educational and awareness programs for the community about hazards of poison exposures along with effective poison information center are considered important strategies for the prevention of these emergencies.

\section{Limitations:}

This study was conducted retrospectively by collecting the data from medical records, which sometimes lacked detailed documentation of cases, especially those referred from other hospitals. In some cases, the chemical nature of the poison was obtained by direct observation of the bottles or packages brought by the patients and/or their relatives. Hence, this information was not always reliable. In addition, only patients presented to PCC-ASUH were included in the study without considering other poison centers, private clinics or patients treated at home. Therefore, the full extent of poisoning in Egypt is likely under-estimated. Finally, the study was conducted over a 1-year period. However, a longer duration would provide greater analysis regarding the problem of poisoning.

\section{References}

Abo El- Noor MM (2013): Pattern of Acute Poisoning among Patients Admitted to Tanta Poison Control Center during 2012. Ain Shams Journal of Forensic Medicine and Clinical Toxicology; 20: 158-169.

Adinew GM and Asrie AB (2016): Pattern of acute poisoning in teaching hospital, northwest Ethiopia. International Journal of Pharmacology and Toxicology; 4 (1): 47-52.

Anthony L and Kulkarni C (2012): Patterns of poisoning and drug overdosage and their outcome among in-patients admitted to the emergency medicine department of a tertiary care hospital. Indian Journal of Critical Care Medicine; 16(3): 130135.

Arun M, Nagesh KR, Vikram P, et al. (2005): Geriatric Poisoning Fatalities: A Manipal Perspective. Indmedica J 2005; 5(1): 01-2005-03.

Asawari R, Atmaram P, Bhagwan K, Priti D, et al. (2017): Toxicological Pattern of Poisoning in Urban Hospitals of Western India. J Young Pharm; 9(3):315-320.

Azab S, Hirshonbc J, Bryan D, et al. (2015): Epidemiology of acute poisoning in children presenting to the poisoning treatment center at Ain Shams University in Cairo, Egypt, 20092013. Clinical Toxicology; 54 (1): 20-26.

Bamathy B, Punnagai K, Amritha C and Chellathai D (2017): Incidence \& Patterns of Acute Poisoning Cases in an Emergency Department of a Tertiary Care Hospital in Chennai. Biomedical \& Pharmacology Journal; 10(3): 1285-1291.

Barratt M, Caki V \& Lenton S, (2013): Patterns of synthetic cannabinoid use in Australia. Drug and alcohol review; 32(2): 141-146.

Cassidy N, Lee SKK, Donegan CF et al. (2008): Poisoning in older adults: the experience of the National Poisons Information Centre. Ir Med J;10: 268-270.

El Masry M and Tawfik H (2013): Annual Report of the Poison Control Centre of Ain Shams University Hospital, Cairo, Egypt. Ain Shams Journal of Forensic Medicine and Clinical Toxicology; 20: 10-17.

Foto-Özdemir D, Akdemir D, and Çuhadaroğlu-Çetin F (2016): Gender differences in defense mechanisms, ways of coping with stress and sense of identity in adolescent suicide attempts. Turkish Journal of Pediatrics; 58(3). 
Gummin DD, Mowry JB, Spyker DA, Brooks DE, et al. (2019): 2018 Annual Report of the American Association of Poison Control Centers' National Poison Data System (NPDS): 36th Annual Report. Clin Toxicol; 57(12): 1220-1413.

Halawa H, Nageeb S, and Guindi M (2013): Annual Report of the Poison Control Centre, Ain Shams University Hospitals, Cairo, Egypt, 2012. Ain Shams Journal of Forensic Medicine and Clinical Toxicology; 21:27-34.

Hassen G, Roy A, Fernandez D, et al. (2018): Analysis of K2 products sold as incense. The American Journal of Emergency Medicine; 36(7): 13071309.

Hegazy MM and Elfiky AK (2016): Pattern of Acute Poisoning Cases Admitted to Menoufia Poisoning and Addiction Control Center: A Prospective Study. Ain Shams Journal of Forensic Medicine and Clinical Toxicology; 26: 35-43.

Huynh A, Cairns R, Brown J, Lynch A, et al. (2018): Patterns of poisoning exposure at different ages: the 2015 annual report of the Australian Poisons Information Centres. Med J; 209(2): 74-79.

Jalali A, Savari M, Dehdardargahi S, and Azarpanah A (2012): The Pattern of Poisoning in Southwestern Region of Iran: Envenoming as the Major Cause. Jundishapur J Nat Pharm Prod; 7(3):100-105.

Karbakhsh M and Zandi NS (2008): Pattern of poisoning in the elderly: an experience from Tehran. Clin Toxicol; 46: 211-217.

Lam (2003): Childhood and adolescence poisoning in NSW, Australia: an analysis of age, sex, geographic, and poison types. Injury Prevention; 9(4): 338-342.

Moghaddam HH, Zamani N, Rahimi M, Shadnia S, et al. (2014): Acute Adult and Adolescent Poisoning in Tehran, Iran; the Epidemiologic Trend between 2006 and 2011. Archives of Iranian Medicine, Volume 17(8): 534- 538.

Mowry JB, Spyker DA, Brooks DE, McMillan N, Schauben JL (2014): 2014 Annual Report of the American Association of Poison Control Centers' National Poison Data System (NPDS): $32^{\text {nd }}$ Annual Report. Clin Toxicol; 53: 962-1147.

Nair PK and Revi NG (2015): One-Year Study on Pattern of Acute Pharmaceutical and Chemical Poisoning Cases Admitted to a Tertiary Care Hospital in Thrissur, India. Asia Pacific Journal of medical toxicology; 2: 79-82.

PCC-ASUH (2016): Poison control Center, Ain Shams University Hospitals, Annual report.

PCC-ASUH (2018): Poison control Center, Ain Shams University Hospitals, Annual report.

Persson HE, Sjoberg GK, Haines JA, and Pronczuk de Garbino J (1998): Poisoning severity score.
Grading of acute poisoning. J Toxicol Clin Toxicol; 36(3):205-213.

Prayag A, Ashtagi GS and Mallapur MD (2016): Pattern of poisoning cases at a tertiary health-care center, Belagavi. International Journal of Medical Science and Public Health; 5(18):1698-1701.

Sahin S, Carman KB and Dinleyici EC (2011): Acute poisoning in children; data of a pediatric emergency unit. Iran J Pediatr; 21(4):479-484.

Sande SD and kumar PU (2017): Pattern of Acute Poisoning Presenting at a Tertiary Care Hospital. IOSR Journal of Pharmacy; 7(9): 39-42.

Schifano F (2014): Misuse and abuse of pregabalin and gabapentin: cause for concern? CNS Drugs; 28(6):491-496.

Shadnia S, Esmaily H, Sasanian G, et al. (2007): Pattern of acute poisoning in Tehran-Iran in 2003. Human \& Experimental Toxicology; 26: 753-756.

Sigdel D, Jha SK, Kathet R, Mainali UK, et al. (2019): Pattern and Outcome of Acute Poisoning Cases in a Tertiary Care Hospital in Eastern Nepal. International Journal of Contemporary Medical Research; 6(4): D27-D31.

Tawfik H and ElHelaly H (2015): Toxicological Profile of Acutely Poisoned Cases Admitted to Poison Control Center, Ain-Shams University Hospitals during Year 2013. Shams Journal of Forensic Medicine and Clinical Toxicology; 24: 154-163.

Tawfik H and Khalifa EA (2017): Evaluation of Poisoning and Drug Overdose among Cases Presented to Poison Control Centre, Ain Shams University Hospital during the Year 2015. Ain Shams Journal of Forensic Medicine and Clinical Toxicology; 29: 100-112.

Thundiyil JG, Stober J, Besbelli N, et al. (2008): Acute pesticide poisoning: a proposed classification tool. Bull World Health Organ; 86:205-209.

Wijegoonawardene I, Kodikara T, Jayaratne SD and Jayawardane P (2015): Pattern of Acute Adult Poisoning at a Tertiary Care Hospital in the Western Province Sri Lanka: A Retrospective Study. Fam Med Med Sci Res; 4:2.

Yuan S, Gao Y, Ji W, et al. (2018): The evaluation of acute physiology and chronic health evaluation II score, poisoning severity score, sequential organ failure assessment score combine with lactate to assess the prognosis of the patients with acute organophosphate pesticide poisoning. Medicine; 97 (21): 1-5.

Zanda M and Fattore L (2018): Old and new synthetic cannabinoids: lessons from animal models. Drug metabolism reviews; 50(1): 54-64. 


\title{
تقييم شدة التعرض للتسمم لاى المرضى الذين تم استقبالهم بمركز علاج التسمم بمستثفيات جامعة عين

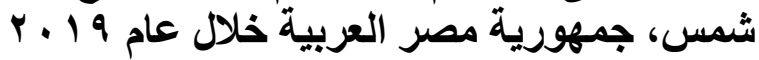

\author{
ولاء جمعة عبد الحميد
}

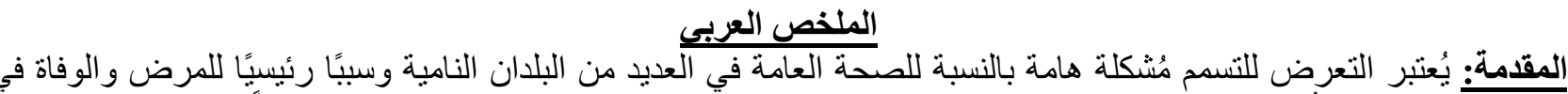

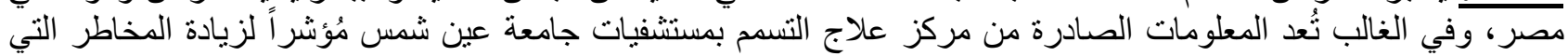
يو اجهوبا المجتمع.

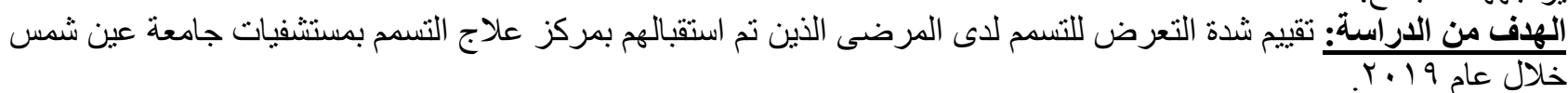

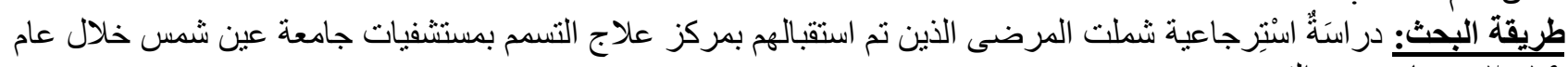

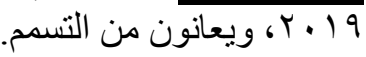

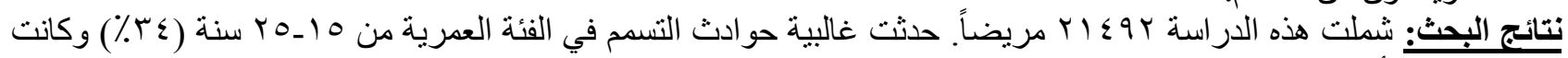

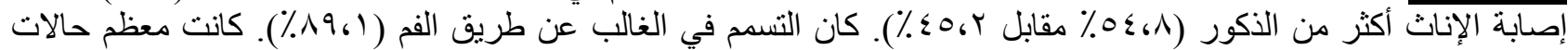

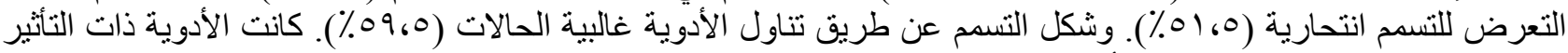

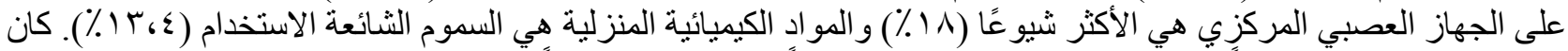

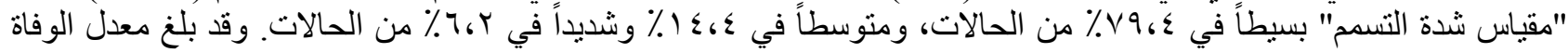

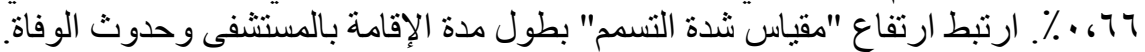

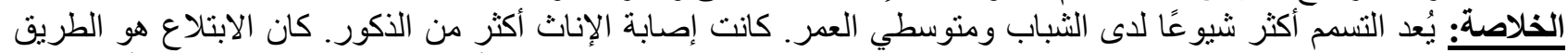

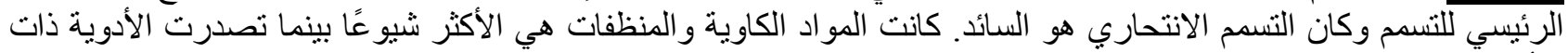

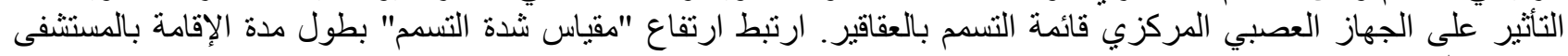
وحدوث الوفاة. التوصيات: يُعتبر "مقياس شدة التسمم" وسيلة بسيطة لتقييم التسمح. نحتاج إلى لو ائح صارمة للتحكم في تسويق الأدوية. 